\title{
Comparison of Level 3 mean monthly GPROF products from GPM and TRMM microwave imager in estimating seasonal precipitation
}

\author{
$\underline{\text { Kaifang Shi }}^{\text {a, b}}$, Yun Chen ${ }^{\mathrm{b}}$ and Jianping $\mathrm{Wu}{ }^{\text {a }}$ \\ ${ }^{a}$ Key Laboratory of Geographic Information Science, Ministry of Education, East China Normal University, \\ Shanghai, China \\ Email:shikf1986@126.com \\ Email:jpwu@geo.ecnu.edu.cn \\ ${ }^{b}$ CSIRO Land and Water Flagship, Canberra, Australia \\ Email:Yun.Chen@csiro.au
}

\begin{abstract}
Reliable and accurate measurement of precipitation plays a critical role in the studies of meteorology, hydrology and water resource management. In the past decades, satellite-based quantitative precipitation products have provided a suitable means to measure precipitation from space. An example is the TRMM (Tropical Rainfall Measuring Mission) microwave imager (TMI) products. It is well known that TMI products have an insensitivity to light precipitation and significant underestimation to heavy precipitation. In early 2014, National Aeronautics and Space Administration (NASA) and Japan Aerospace Exploration Agency (JAXA) successfully launched a new generation of satellite, Global Precipitation Measurement (GPM) Core Observatory. Onboard the GPM core satellite has a multichannel GPM microwave imager (GMI) which uses 13 different microwave channels to observe energy from the different types of precipitation through clouds for estimating everything from low to high precipitation. At present, several composite precipitation products with algorithms that related GMI and partner passive microwave sensors have been released, including Level 2 gridded Goddard Profiling GMI (2GPROF-GMI) and Level 3 mean monthly GPROF-GMI (3GPROF-GMI) products. It is therefore important to investigate whether these new GMI products are more reliable in estimating precipitation than those of TMI. In this study, differences between 3GPROF-GMI and 3GPROF-TMI products were compared over different surface types on a nearglobal scale for different seasons. The results show that a systematic difference (3GPROF-GMI $>3$ GPROFTMI) for low and high precipitation. High positive correlation coefficient (CC) values are mainly in the Caribbean region, South Atlantic and over the African continent. Low CC values concentrate in equatorial regions, North Atlantic and Northwest Pacific. In addition, high mean absolute difference (MAD) and root mean square difference (RMSD) values dominate over the Tropics. By contrast, low negative MAD and RMSD values are found in the Inter-Tropical Convergence Zone (ITCZ). Histograms of both products are very similar; however higher frequencies for 3GPROF-GMI are found in the low and high precipitation ranges than those of 3GPROF-TMI. Statistics for different precipitation ranges reveal more details on the systematic differences over land and ocean. For low and relatively low precipitation, the MAD and RMSD values over land are slightly lower than those over oceans; on the other hand, the CC values over land are larger than those over oceans. For medium precipitation, the MAD, RMSD and CC values over oceans are in general higher than those over land in all seasons; but for relatively high precipitation, the opposite is found. The results of this study enhance the understanding of the difference between these two products, and provide a viable validation analysis for the updated GMI products.
\end{abstract}

Keywords: $\quad$ Precipitation, Comparison, GPM, TRMM, GPROF 


\section{INTRODUCTION}

Reliable quantitative estimates of regional and global precipitation are critical for the studies of meteorology, hydrology and water resource management (Liu, 2015; Sahoo et al., 2015). However, accurate estimation of precipitation remains a challenge, especially in data sparse areas (Chen et al., 2013a). In the past decades, satellite-based quantitative precipitation products have provided an efficient way to measure precipitation from space (Chen et al., 2013b; Mahmud et al., 2015). Recently, the Tropical Rainfall Measuring Mission (TRMM) microwave imager (TMI) products, developed with algorithms that utilize multi-satellites and multi-sensors, have been widely used in meteorological and hydrological research. Yet it is well known that TMI products have an insensitivity to light precipitation and heavy precipitation (Prakash et al., 2013). In early 2014, the National Aeronautics and Space Administration (NASA) and the Japan Aerospace Exploration Agency (JAXA) successfully launched a new generation of satellite, the Global Precipitation Measurement (GPM) Core Observatory. Onboard the GPM core satellite has a multichannel GPM microwave imager (GMI) which uses 13 different microwave channels to observe energy from the different types of precipitation through clouds for estimating everything from low to high precipitation (Draper et al., 2015a; Draper et al., 2015b). At present, several composite precipitation products with algorithms related to GMI and partner passive microwave sensors have been released, including Level 2 gridded Goddard Profiling GMI (2GPROF-GMI) and Level 3 mean monthly GPROF-GMI (3GPROF-GMI) products. It is therefore important to investigate whether these new GMI products are more reliable in estimating precipitation than those of TMI. Based on current data availability at near global scale, we take 3GPROFGMI and 3GPROF-TMI products as examples to explore their monthly differences over different surface types on a near-global scale.

\section{DATA AND METHODS}

Both 3GPROF-GMI and 3GPROF-TMI products are downloaded from the website of GPM-NASA (http://pmm.nasa.gov/data-access/downloads/gpm). They have the same temporal resolution (monthly), spatial resolution $(0.25 \times 0.25$ degree) and date range (March, $2014-$ February, 2015). Four seasons, spring (March, April and May, MAM hereafter), summer (June, July and August, JJA hereafter), autumn (September, October and November, SON hereafter) and winter (December, January and February, DJF hereafter) were considered. Moreover, land and ocean were separated in computing statistics. Different precipitation ranges were also taken into account. They were defined as: low precipitation $(\mathrm{LP}, 0.01<\mathrm{mm} / \mathrm{h})$, relatively low precipitation (RLP, 0.01-0.25 mm/h), medium precipitation (MP, 0.25-0.75 $\mathrm{mm} / \mathrm{h}$ ) and relatively high precipitation (RHP, $>0.75 \mathrm{~mm} / \mathrm{h}$ ). Note that the American Meteorological Society has different thresholds for daily precipitation classifications (http://glossary.ametsoc.org/wiki/Rain); nonetheless, the purpose here is to investigate monthly statistical variations in different precipitation ranges. Mean absolute difference (MAD), root mean square difference (RMSD) and correlation coefficient (CC) (Ebert, 2007) were also used to measure differences between the two products.

\section{RESULTS}

\subsection{Seasonal comparison at near-global scale}

The 3 GPROF-GMI and $3 \mathrm{GPROF}-\mathrm{TMI}\left(40^{\circ} \mathrm{N}-40^{\circ} \mathrm{S}, 180^{\circ} \mathrm{E}-180^{\circ} \mathrm{W}\right)$ average seasonal precipitation estimated between March, 2014 and February, 2015 are illustrated in Figure 1. Both estimates qualitatively show similar large-scale patterns indicating that precipitation mainly falls in the Tropics $\left(10^{\circ} \mathrm{N}-10^{\circ} \mathrm{S}, 180^{\circ} \mathrm{E}-\right.$ $180^{\circ} \mathrm{W}$ ), especially over tropic ocean. However, there are obvious differences in magnitude over the high precipitation range, like over the Indian Ocean (" $n$ " in Figure 1c) and the Amazon Rain forest ("m" in Figure 1c). Especially in JJA and SON, the 3GPROF-GMI product overestimates precipitation compared to 3GPROF-TMI by about $0.75 \mathrm{~mm} / \mathrm{h}$ over the Indian Ocean. Four typical scatterplots for different season are shown in Figure 2 to further demonstrate the difference between 3GPROF-GMI and 3GPROF-TMI products. The linear fit lines are below the 1:1 line and the intercept values are above zero in all seasons, indicating a systematic difference (3GPROF-GMI > 3GPROF-TMI) in light rain events (Liu, 2015). Moreover, 3GPROFGMI has more points of higher precipitation than 3GPROF-TMI (Figure 2), which is consistent with the finding in Figure 1. 
Figure 3 consists of four mean seasonal CC maps. Overall, high positive CC values dominate in the Caribbean region ("m" in Figure 3a), over the South Atlantic ("n"in Figure 3a) and over the African continent. The possible cause of the similarity is driven by the low precipitation in these areas. Low CC values concentrate in equatorial regions, the North Atlantic (" $k$ " in Figure 3a) and the Northwest Pacific ("i" in Figure 3a). All the negative $\mathrm{CC}$ values are scattered in various places, such as the Indian Ocean and North Atlantic. These low points perhaps are related to the uncertainty in low and high precipitation estimation as mentioned earlier. In short, despite the change in data sources, precipitation rates in major regions maintain a

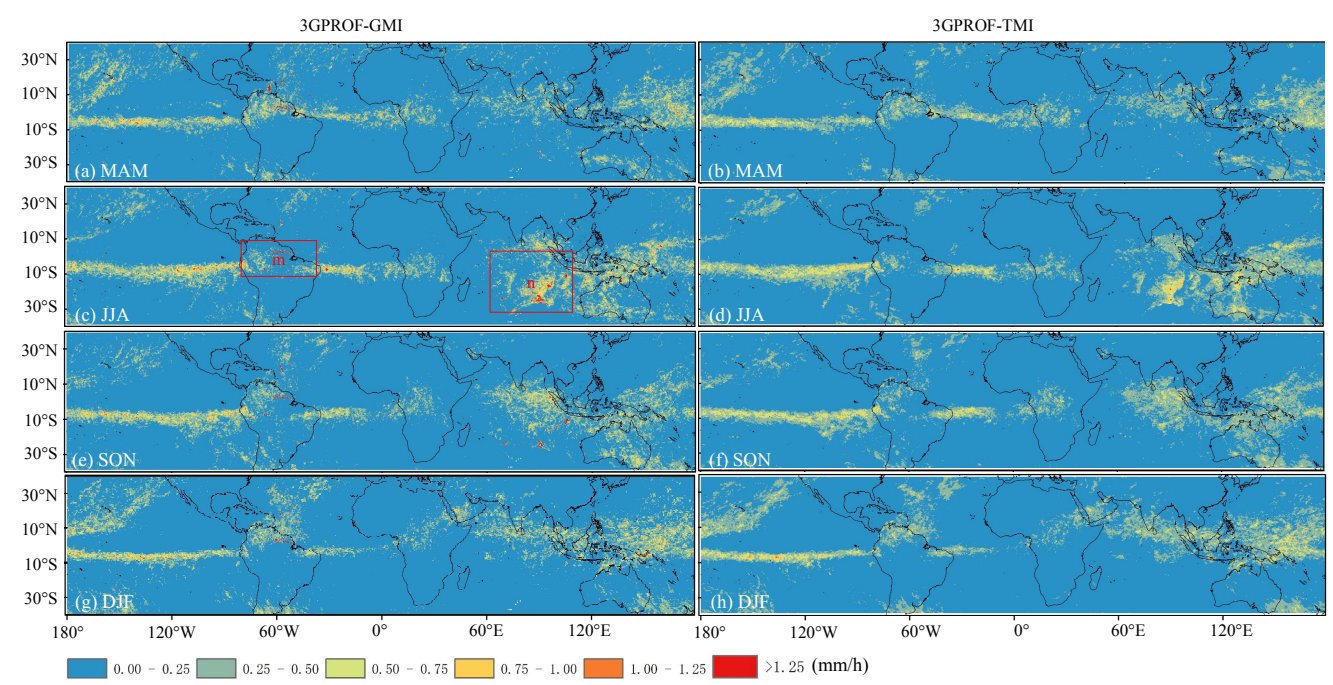

Figure 1. Seasonal monthly mean precipitation rate.

good linear relationship in all seasons.

Figure 4 shows four mean seasonal MAD maps. In general, high MAD values dominate over Tropics, especially in India Ocean and Amazon Rain Forest of JJA and SON. By contrast, low negative MAD values are found over land and ocean in the Inter-Tropical Convergence Zone (ITCZ) $\left(30^{\circ} \mathrm{N}-40^{\circ} \mathrm{N}, 180^{\circ} \mathrm{E}-\right.$ $\left.180^{\circ} \mathrm{W} ; 30^{\circ} \mathrm{S}-40^{\circ} \mathrm{S}, 180^{\circ} \mathrm{E}-180^{\circ} \mathrm{W}\right)$. Like MAD, RMSD in Figure 5 presents more consistent patterns in all seasons. RMSD values are low in ITCZ, and high in Tropics.

Figure 6 are the histograms of precipitation rate frequencies for the four seasons. The distribution patterns of both products are very similar with highest frequencies in the medium precipitation regime. However, for the low and high precipitation, 3GPROF-TMI is not as high as in 3GPROF-GMI, which is consistent with the findings in Figure 2.

\subsection{Seasonal comparison between land and ocean}

The scatter plots of seasonal monthly mean precipitation rate over land and ocean are shown in Figure 7. The distribution and magnitudes of precipitation rate can vary with seasons between different surface types (Figure 7). There are also some false alarm samples along both 3GPROF-GMI and 3GPROF-TMI axes, mainly in the LP, where no precipitation is presented in 3GPROF-TMI but precipitation found in 3GPROFGMI, and vice versa. All linear fit lines that are below the 1:1 line indicates that a significant difference in light rain events, which is consistent with the finding in Figure 1 and 2.

Table 1 shows the inter-seasonal variation of MAD, RMSD and CC values over land and oceans for precipitation ranges from LP to RHP. For LP and RLP, the MAD and RMSD values over land are slightly smaller than those over oceans; oppositely, the $\mathrm{CC}$ values over land are larger than those over oceans. For MP, the MAD, RMSD and CC values over oceans are in general larger than those over land in all seasons; 


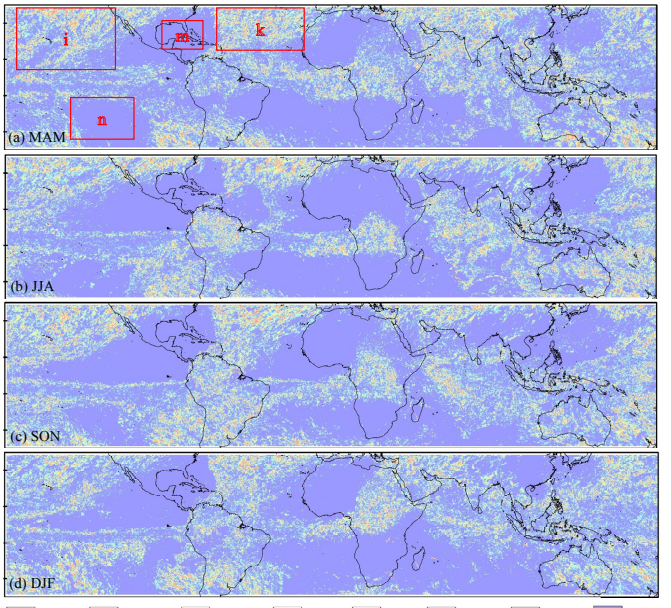

Figure 3. $\mathrm{CC}$ of seasonal mean precipitation rate.

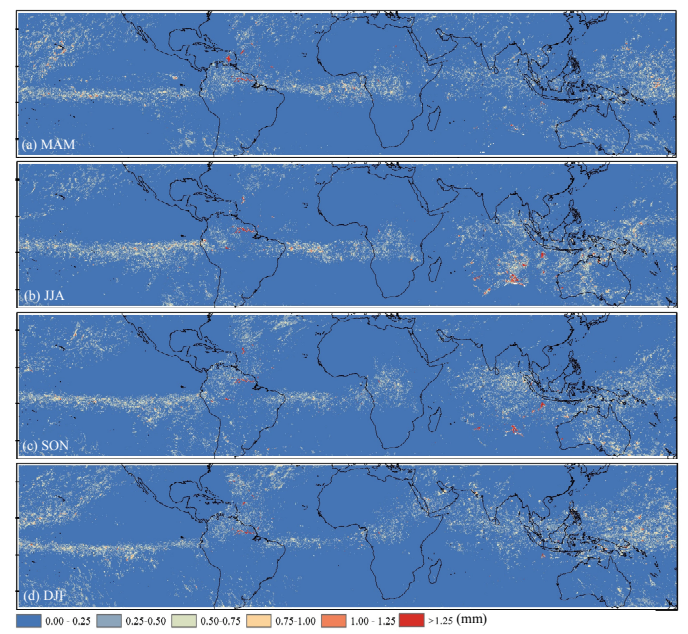

Figure 5. RMSD of seasonal mean precipitation rate.

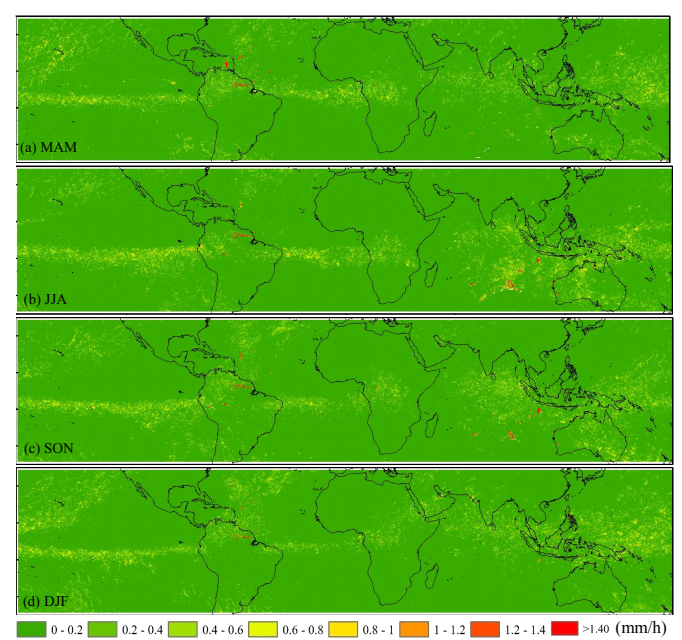

Figure 4. MAD of seasonal mean precipitation rate.

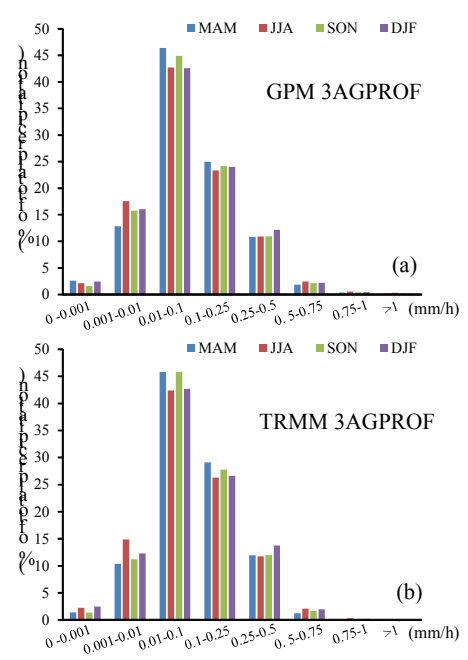

Figure 6. Histograms of seasonal mean precipitation rate.

but for RHP, the opposite is found. These different results between land and oceans may be contributed by many factors, such precipitation magnitude, topography, and vegetative cover.

\section{CONCLUSION}

This study examines the differences between 3GPROF-GMI and 3GPROF-TMI products in different seasons and precipitation ranges over different surface types on a near global scale. Scatter plots suggests a systematic difference (3GPROF-GMI > 3GPROF-TMI) for low and high precipitation. High positive CC values dominate in Caribbean region, South Atlantic and African continent, and Low CC values concentrate in equatorial regions, North Atlantic and Northwest Pacific. In addition, high MAD and RMSD values dominate over Tropics. By contrast, low negative MAD and RMSD values are found in ITCZ. Histograms of both products are very similar; however higher frequencies for 3GPROF-GMI are found in the low and high precipitation ranges than those in 3GPROF-TMI. Statistics for different precipitation ranges reveal more details on the systematic differences. For LP and RLP, the MAD and RMSD values over land are slightly lower than those over oceans; on the other hand, the CC values over land are higher than those over oceans. For MP, the MAD, RMSD and CC values over oceans are in general higher than those over land in all seasons; but for RHP, the opposite is found. The difference in the range of microwave channels is probably 

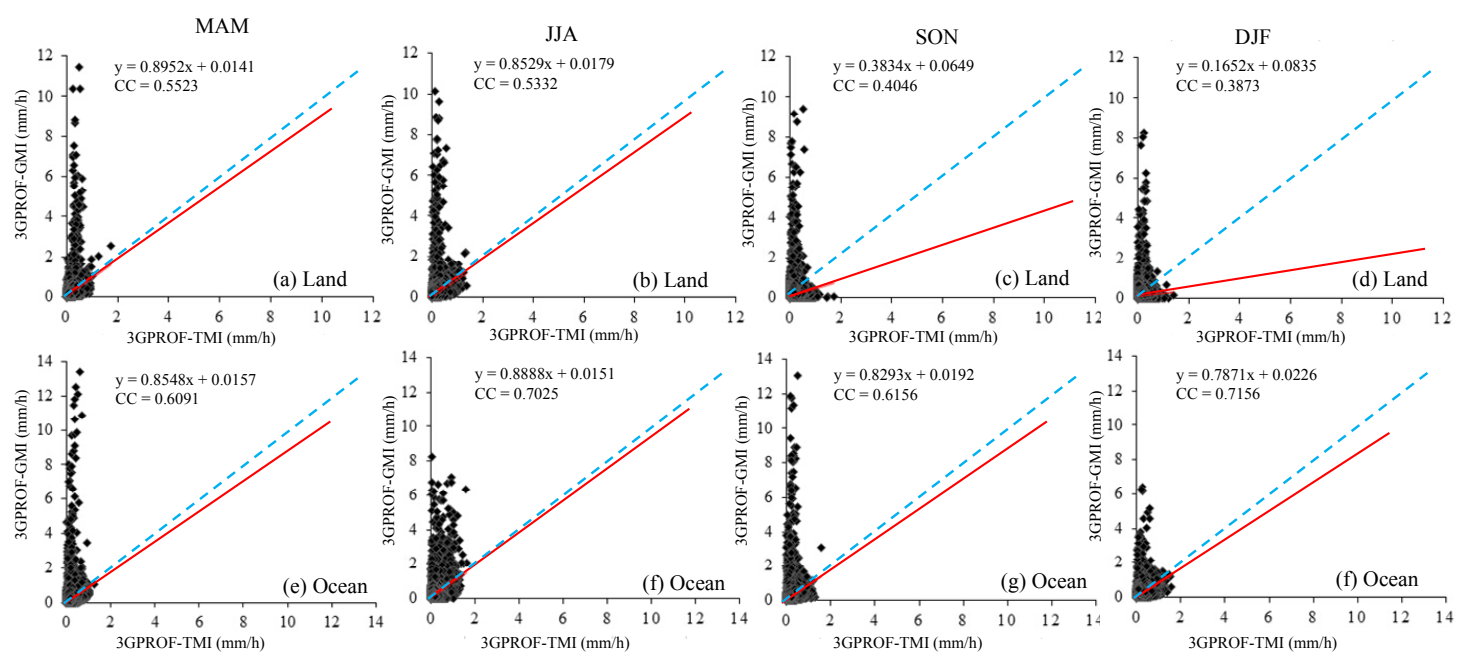

Figure 7. Scatter plots of seasonal monthly mean precipitation rate over land and ocean.

Table 1. MAD, RMSD, and CC of seasonal monthly mean precipitation rate within different precipitation ranges over land and ocean.

\begin{tabular}{|c|c|c|c|c|c|c|c|c|c|c|c|c|c|}
\hline \multirow{2}{*}{$\begin{array}{l}\text { Land } \\
\text { type }\end{array}$} & \multirow{2}{*}{ Season } & \multicolumn{3}{|c|}{ LP } & \multicolumn{3}{|c|}{ RLP } & \multicolumn{3}{|c|}{ MP } & \multicolumn{3}{|c|}{ RHP } \\
\hline & & MAD & RMSD & $\mathrm{CC}$ & MAD & RMSD & $\mathrm{CC}$ & MAD & RMSD & $\mathrm{CC}$ & MAE & RMSD & $\mathrm{CC}$ \\
\hline \multirow{3}{*}{ Land } & MAM & 0.010 & 0.014 & 0.971 & 0.068 & 0.086 & 0.585 & 0.220 & 0.276 & 0.643 & 1.276 & 1.387 & 0.738 \\
\hline & JJA & 0.005 & 0.007 & 0.990 & 0.069 & 0.086 & 0.562 & 0.225 & 0.282 & 0.626 & 1.181 & 1.351 & 0.625 \\
\hline & DJF & 0.007 & 0.010 & 0.986 & 0.070 & 0.088 & 0.592 & 0.214 & 0.269 & 0.638 & 0.609 & 1.163 & 0.687 \\
\hline \multirow{2}{*}{ Ocean } & MAM & 0.012 & 0.016 & 0.970 & 0.076 & 0.096 & 0.554 & 0.223 & 0.280 & 0.683 & 0.813 & 1.018 & 0.750 \\
\hline & DJF & 0.009 & 0.012 & 0.979 & 0.078 & 0.098 & 0.589 & 0.223 & 0.276 & 0.690 & 0.967 & 0.767 & 0.770 \\
\hline
\end{tabular}

one of the main reasons why 3GPROF-GMI products have more accurate perception estimation than those of 3GPROF-TMI. We expect that the constantly updated GPM- GMI products provide a foundation for forecasting and accurate precipitation around world. Overall, this study on the differences of 3GPROF-GMI and 3GPROF-TMI products provide a useful feedback to scientific researchers for making a viable validation analysis for the future GMI products. Due to the limitation of data availability for 2014-2015, there is a lack of discussion about the comparison between the two products and ground observations. Ongoing research is investigating the mechanisms of the difference between 3GPROF-GMI and 3GPROF-TMI products using global rainfall data from gauging stations.

\section{ACKNOWLEDGMENTS}

This work is supported by the China Scholarship Council (No. 201406140007).

\section{REFERENCES}

Chen, S., Y. Hong, Q. Cao, J.J. Gourley, P.E. Kirstetter, B. Yong, Y. Tian, Z. Zhang, Y. Shen, and J. Hu. (2013a). Similarity and difference of the two successive V6 and V7 TRMM multi-satellite precipitation analysis performance over China. Journal of Geophysical Research: Atmospheres, 118, 13060-13074.

Chen, S., Y. Hong, J.J. Gourley, G.J. Huffman, Y. Tian, Q. Cao, B. Yong, P.E. Kirstetter, J. Hu, and J. Hardy. (2013b). Evaluation of the successive V6 and V7 TRMM multi-satellite precipitation analysis over the Continental United States. Water Resources Research, 49, 8174-8186.

Draper, D.W., D. Newell, D.S. McKague, and J.R. Piepmeier. (2015a). Assessing calibration stability using the Global Precipitation Measurement (GPM) Microwave Imager (GMI) noise diodes. IEEE Journal of Selected Topics in Applied Earth Observations and Remote Sensing, Online first. 10.1109/JSTARS.2015.2406661. 
Draper, D.W., D. Newell, F.J. Wentz, S. Krimchansky, and G.M. Skofronick-Jackson. (2015b). The Global Precipitation Measurement (GPM) Microwave Imager (GMI): instrument overview and early on-orbit performance. IEEE Journal of Selected Topics in Applied Earth Observations and Remote Sensing, Online first. 10.1109/JSTARS.2015.2403303.

Ebert, E.E. (2007). Methods for verifying satellite precipitation estimates. Measuring precipitation from space. Advances in Global Change Research, 28, 345-356.

Liu, Z. (2015). Comparison of versions 6 and 7 3-hourly TRMM multi-satellite precipitation analysis (TMPA) research products. Atmospheric Research, 163, 91-101.

Mahmud, M.R., S. Numata, H. Matsuyama, T. Hosaka, and M. Hashim. (2015). Assessment of effective seasonal downscaling of TRMM precipitation data in Peninsular Malaysia. Remote Sensing, 7, 4092-4111.

Prakash, S., C. Mahesh, and R. Gairola. (2013). Comparison of TRMM multi-satellite precipitation analysis (TMPA)-3B43 version 6 and 7 products with rain gauge data from ocean buoys. Remote Sensing Letters, 4, 677-685.

Sahoo, A.K., J. Sheffield, M. Pan, and E.F. Wood. (2015). Evaluation of the Tropical Rainfall Measuring Mission Multi-Satellite Precipitation Analysis (TMPA) for assessment of large-scale meteorological drought. Remote Sensing of Environment, 159, 181-193. 